\title{
Enjeux politiques, didactiques et culturels de la méthode directe en Algérie, pour l'enseignement du français, du kabyle et de l'arabe (1880-1920)
}

Political, didactic and cultural issues of the direct method in Algeria, for the teaching of French, Kabyle and Arabic (1880-1920)

Nadia Berdous et Claude Cortier

\section{OpenEdition}

Journals

\section{Édition électronique}

URL : https://journals.openedition.org/dhfles/8004

DOI : $10.4000 /$ dhfles. 8004

ISSN : 2221-4038

\section{Éditeur}

Société Internationale pour l'Histoire du Français Langue Étrangère ou Seconde

\section{Édition imprimée}

Date de publication : 1 décembre 2020

Pagination : $259-279$

ISSN : 0992-7654

Référence électronique

Nadia Berdous et Claude Cortier, «Enjeux politiques, didactiques et culturels de la méthode directe en Algérie, pour l'enseignement du français, du kabyle et de l'arabe (1880-1920) », Documents pour I'histoire du français langue étrangère ou seconde [En ligne], 64-65 | 2020, mis en ligne le 10 mars 2021, consulté le 25 mars 2023. URL : http://journals.openedition.org/dhfles/8004 ; DOI : https://doi.org/ $10.4000 /$ dhfles. 8004

Ce document a été généré automatiquement le 25 mars 2023.

Tous droits réservés 


\section{Enjeux politiques, didactiques et culturels de la méthode directe en Algérie, pour l'enseignement du français, du kabyle et de l'arabe (1880-1920)}

Political, didactic and cultural issues of the direct method in Algeria, for the teaching of French, Kabyle and Arabic (1880-1920)

Nadia Berdous et Claude Cortier

\section{Introduction}

1 La fin de l'Empire et les débuts de la $\mathrm{III}^{\mathrm{e}}$ République marquent la fin d'une politique arabo-française menée par Napoléon III, accordant une place importante à une langue arabe modernisée, dans le cadre d'un projet de nation algéro-française moderne (Messaoudi 2014: 80). L'expansion de la population européenne et la colonisation agricole en développement engagent les autorités à considérer comme une priorité la francisation des immigrants espagnols, italiens et maltais de la colonie.

2 L'application des lois scolaires de Jules Ferry à l'Algérie (décret du 13 février 1883) va instaurer un projet d'instruction concerté, touchant principalement les villes et la Kabylie. De nombreux textes ou rapports émanant de hauts fonctionnaires de l'Instruction publique tels que Louis Machuel, Pierre Foncin, Alfred Rambaud, Marcellin Berthelot, Léon Bourgeois, et plus tard Ferdinand Buisson et les auteurs du Dictionnaire de pédagogie et d'instruction primaire (1887) témoignent d'une volonté d'instruire les populations autochtones et de les conduire à la civilisation française. Le recteur Charles Jeanmaire, appuyé par le ministre Ferry et l'Alliance française (Cortier 1998), parviendra durant son mandat (1884-1908), à poser les bases du développement 
scolaire des populations indigènes ${ }^{1}$ et d'une assimilation par l'école, avant d'être rappelé en France, sous la pression des colons algériens. Cette politique va s'appuyer sur des hommes de terrain parlant l'arabe et le kabyle, ainsi que sur le rôle prépondérant des institutions de formation, notamment l'École normale de Bouzareah puis l'École supérieure des Lettres d'Alger pour la formation des enseignants et l'élaboration du matériel pédagogique.

3 L'enseignement des langues autochtones (arabe, berbère, kabyle) a été pensé dans l'objectif du développement de la mission civilisatrice, afin de faciliter le rapprochement des deux principaux types de population, les colons, pour la plupart d'origine européenne et les 'indigènes', populations de traditions berbères ou arabes. Il s'agissait aussi de former des cadres intermédiaires indigènes connaissant le français et des cadres européens connaissant l'arabe et/ou le berbère pour pouvoir exercer dans des régions rurales.

4 Notre article souhaite montrer comment ces volontés politiques, confrontées à certaines urgences, vont se conjuguer aux apports des enseignants initiés en France aux pédagogies nouvelles pour créer des manuels qui fourniront un terrain propice aux développements de la méthode directe (désormais $\mathrm{MD}$ ). Les recommandations officielles, qui se multiplieront dans les années 1880-1900, en faveur de la MD contribueront à en diffuser, sinon la stricte méthodologie, du moins l'esprit, dans la plupart des manuels publiés dans cette période. Car tous les pédagogues républicains soulignent la nécessité d'adapter contenus d'enseignement et méthodes à la «mentalité des indigènes ", pour allier pratique et concret, en s'attachant aux réalités et en développant le «bon sens critique » (Poulard $1910: 33$ ).

\section{Développement de l'instruction et priorité accordée au français}

\section{Organisation de l'enseignement et priorité au français}

Lorsque en 1883, la décision est prise par le gouvernement français d'étendre les lois scolaires à l'Algérie, de supprimer les écoles primaires arabes-françaises et de scolariser en français les jeunes Algériens, avec les mêmes programmes que les enfants français, l'ambition du législateur est claire : «combler la distance » entre la France et l'Algérie, « civiliser ». Dans un premier temps, grâce au recteur Jeanmaire, l'école mise en place en Algérie et financée en partie par l'État, tentera de prendre ses distances à l'égard des colons, ce qui lui conférera une certaine légitimité auprès des populations musulmanes. Les colons sont en effet partisans pour celles-ci d'une école strictement professionnelle tandis que les Républicains souhaitent une école tournée vers une formation plus générale (Mariet 1978 : 429).

Seront organisés principalement deux types d'école (sans séparation absolue): les écoles pour les "Européens » et les écoles pour les «Indigènes ", où l'arabe n'occupera plus qu'un espace restreint, sous la forme d'un enseignement de la langue usuelle. Les enfants 'indigènes' sont admis au même titre que les Européens « aux conditions fixées par les lois et règlements dans les écoles publiques de tout degré ", "la liberté de conscience des élèves est formellement garantie » (Jeanmaire 1911). 
7 Dans ces écoles, la plus grande partie de la classe est consacrée à l'enseignement du français (13 heures par semaine au cours préparatoire, $11 \mathrm{~h} 30$ au cours élémentaire) selon une progression spécifique: l'étude du français ne reste pas cantonnée sur quelques exercices spéciaux, elle résulte de toutes les leçons de l'école (Musée pédagogique 1890).

\section{Le rôle de Jean-Eugène Scheer pour le développement de la méthode directe}

8 En 1881, Eugène Scheer, instituteur d'origine alsacienne, parlant l'arabe et le kabyle, fut chargé d'organiser le développement de l'enseignement public en Algérie, en particulier en Kabylie. Il s'occupa d'abord de la construction des bâtiments, du recrutement des maîtres et mit au point, avec la collaboration du dessinateur Charles Mailhes, une Méthode de lecture-écriture à l'usage des indigènes et des kabyles (1884). Cette méthode sera couramment utilisée et unanimement appréciée (Buisson $1887: 496$ ), car elle s'appuie sur les connaissances de l'élève et son milieu, au contraire des manuels français dont celui de Carré, où les descriptions des objets tels que «matelas, lampadaire, divan, piano " et autres proposent des modèles inconnus aux élèves. "Le livre ne s'adresse pas du tout aux indigènes, malgré les promesses du titre » (Bisquerra $2001: 167)$.

Inspecteur des écoles indigènes, Scheer contribua ensuite à la rédaction des programmes. Il y insiste fortement sur le rôle des exercices de langage, recommande que l'apprentissage du français soit fait directement sans grammaire «selon la méthode par laquelle les mères apprennent à leurs enfants »: "Il exigeait qu'on ne prononçât pas un seul mot nouveau sans l'expliquer et sans montrer au besoin la chose correspondante. Toute classe devait avoir son musée scolaire» (Rambaud $1887: 518$ ). La MD ainsi proposée, correspondant au modèle adopté dans la plupart des colonies (Vigner 2018 : 177) va figurer dans les programmes algériens et les exercices de langage occuperont neuf heures par semaine au cours préparatoire.

10 Suite à son décès prématuré, Alfred Rambaud dans la Revue pédagogique lui consacra un article d'hommage sous le titre " Nos pionniers en Afrique ", soulignant sa ténacité, son abnégation et l'importance de son rôle pour donner à l'enseignement son organisation, ses programmes, ses premiers livres et former son personnel. (Rambaud 1887 :522)

\section{Formation des maîtres à l'ENS de Bouzareah : un pôle actif d'enseignement de l'arabe et du kabyle}

11 Une préparation spéciale des maîtres fut organisée à l'École normale de Bouzareah, celle des instituteurs français dans une section spéciale, et celle des maîtres indigènes dans un cours normal.

Les élèves de la Section spéciale étaient recrutés parmi les instituteurs français ayant déjà exercé des fonctions d'enseignement, ou, à défaut, titulaires du brevet supérieur ou du brevet élémentaire. Les études duraient un an, avec un programme spécifique comprenant la pédagogie spéciale pour l'enseignement des indigènes, l'agriculture, le travail manuel, l'hygiène et la médecine usuelle, les langues arabe et kabyle et les mœurs et coutumes des indigènes de l'Algérie. 

kabyles pourvus du certificat d'études primaire. Ils y complétaient durant quatre ans leur instruction et s'exerçaient à la pratique dans la classe indigène de l'école annexe, selon une pédagogie fondée sur l'observation et la démonstration expérimentale. Le brevet supérieur comportait également des épreuves d'arabe (version et entretien). Tous les anciens élèves, professeurs et directeurs s'accordent à dire que cette école normale fut un véritable laboratoire pédagogique, pour le français mais aussi pour l'arabe et le kabyle.

\section{La méthode directe et l'enseignement des langues maternelles en Algérie coloniale}

\section{Écoles arabes-françaises et innovations dans l'enseignement des langues}

14 Selon Larzul et Messaoudi, qui ont analysé les manuels publiés au XIX ${ }^{e}$ siècle, la MD aurait connu un premier développement dans le cadre de l'enseignement du français aux élèves des écoles arabes-françaises ${ }^{3}$ avec des méthodes innovantes " pour initier les élèves à la modernité européenne. Prenant en charge une double culture, ces textes n'en reflètent pas moins l'adhésion de leurs auteurs, souvent proches des saintsimoniens, au projet colonial » (Larzul 2014 :72).

(ette organisation bilingue des études fut abandonnée, du fait de l'écart entre langue arabe littéraire et langue usuelle, pour donner la primauté au français. On choisit une méthode où l'apprentissage du français ne nécessitait plus l'intermédiaire de l'arabe, "en prenant pour modèle celle qui était employée en Alsace pour l'enseignement du français aux enfants qui ne connaissaient que le 'patois allemand' [...]. Pour ce faire, la direction du collège avait acquis la méthode des tableaux et images publiée par la maison Fasoli à Strasbourg ${ }^{4} »$ (Messaoudi 2014 : 96).

16 Les manuels publiés en Algérie après 1870 sont destinés à un public diversifié, intégrant différentes variétés de l'arabe et des contenus variés (dialogues et proverbes). Ils sont composés de leçons qui suivent une progression grammaticale, abordant les points spécifiques à l'arabe, du simple au complexe. La leçon est basée sur un texte court suivi d'exercices d'application. Les trois principaux ouvrages de ce type sont : -MACHUEL, Louis (1875). Méthode pour l'étude de l'arabe parlé (idiome algérien). Alger: Jourdan.

18 -MACHUEL, Louis (1877). Première année d'arabe. Alger : Jourdan.

19 -BEN SEDIRA, Belkassem (1875). Cours pratique de langue arabe à l'usage des écoles d'Algérie. Exercices d'écriture, de lecture et de mémoire; règles du langage, thèmes et versions; dialogues variés, contes amusants, lettres familières. Alger : Jourdan.

Dans sa préface, Ben Sedira exprime sa dette envers les « leçons pédagogiques » qu'il a reçues à l'École normale de Versailles et les méthodes des germanistes ou anglicistes côtoyés à Paris. Les trois ouvrages accordent une grande part à la langue orale :

21 - «dialogues renfermant des formules de salutations, des compliments », " un grand nombre de proverbes », « une liste d'idiotismes, locutions familières, classées par ordre 
alphabétique », « des listes de mots souvent employés dans la conversation » (Machuel [1875] 1900 : V ; VII et sv.).

22 - des exercices pour habituer l'oreille à distinguer des consonnes inusitées en français et des dialogues destinés à exercer l'expression orale, mais aussi des contes et des fables qui seront dictés en classe, analysés et appris de mémoire, pour servir de matière à un questionnaire oral ou écrit (Ben Sedira 1900 [1875]).

Ces manuels s'appuient sur des «enquêtes» de langue parlée réalisées par leurs auteurs ou leurs prédécesseurs ${ }^{5}$ et valorisent l'arabe parlé ou «vulgaire »: «Il est d'ailleurs injuste et impropre de qualifier dédaigneusement de vulgaire, une langue aussi pittoresque, qui est parlée par un aussi grand nombre d'individus de tous rangs et de toutes classes " (Ben Sedira : VII, 1886).

Ben Sedira a élaboré également des manuels pour l'enseignement du kabyle à l'École des Lettres d'Alger. Son Cours de langue kabyle, grammaire et versions (1887), est un manuel volumineux de 600 pages, comportant de nombreux morceaux choisis collectés en Kabylie.

\section{De l'arabe parlé à la méthode directe : dialectologie et pédagogie}

À partir de 1888, des primes furent instaurées pour encourager les instituteurs européens à entreprendre l'apprentissage de l'arabe parlé et du kabyle (300 francs par an pour les brevets et 500 francs pour les diplômés). Cet enseignement qui concerna d'abord les instituteurs nommés dans les régions arabophones ou berbérophones, fut élargi ensuite à tous les fonctionnaires souhaitant bénéficier de la prime. Les enquêtes et publications d'ordre linguistique et pédagogique vont alors se multiplier, encouragées par des professeurs de l'École des Lettres d'Alger renommés, comme René Basset (éminent spécialiste de langues berbères) et vont alimenter les cours et les manuels.

En 1891, Gaétan Delphin, titulaire de la chaire d'arabe à Oran, publie un Recueil de textes pour l'étude de l'arabe parlé (in-16 $6^{\circ}$ VI-367 p.), collectés autour de Mascara auprès des «Bédouins » et explique dans la préface qu'il a pu «sténographier leurs paroles ». Ce sont ensuite les travaux de William Marçais qui feront autorité avec Le dialecte arabe parlé à Tlemcen: grammaire, textes et glossaire (1902) constitué de poésies et chansons, berceuses, contes recueillis auprès d'un grand nombre d'informateurs locaux. Dans cet ouvrage, "les faits linguistiques sont exposés avec la plus grande minutie et les mécanismes du parler établis. [...] et c'est grâce à l'apport de la dialectologie que les ouvrages élémentaires d'arabe algérien sont largement renouvelés au $\mathrm{XX}^{\mathrm{e}}$ siècle.» (Larzul 2010 : 97)

27 Le manuel de Joseph Desparmet Enseignement de l'arabe dialectal d'après la méthode directe (2 vol. 1904-1905; réédition vocalisée en 1907-1913), s'inscrit dans cette veine car il est " fondé sur le parler de Blida, associant les mots et les choses ». Avec la MD il propose, " par un apprentissage vivant et oral de la langue, une connaissance concrète des 'Coutumes, institutions, croyances ${ }^{6}$ " (Messaoudi $2015: 142$ ).

L'ouvrage inclut une Note sur les dialectes algériens, répertoriant les publications susceptibles d'être utiles aux maitres d'autres régions. À l'exception de la préface, l'ensemble du texte est en arabe, alternant dans la première partie caractères latins et arabes. Desparmet innove tout particulièrement sur le plan de la transcription orale et 
de la vocalisation. «L'arabe dialectal [étant] en vogue », il n'est pas possible de laisser une partie aussi importante que la prononciation à l'empirisme " (Desparmet 1907 : XII). Il critique ses prédécesseurs dont «les textes d'arabe vulgaire ne comportent aucune voyelle ", sous prétexte qu'elles ne sont pas stabilisées, à la différence des «recueils d'arabe régulier soigneusement vocalisés». Mais, "en s'abstenant de marquer les voyelles dans un texte d'arabe vulgaire, on met le lecteur dans la nécessité, non seulement de deviner les voyelles, mais leur place et même les syllabes... » (ibid.). C'est pourquoi il créera un système de vocalisation adapté « emprunté aux chanteurs indigènes, dépositaires de la littérature populaire » (ibid. : IX) pour faciliter la lecture.

De nombreux chants et poésies, historiettes, devinettes, puisées dans la littérature orale servent dans ce manuel de textes pour l'apprentissage, contestant de fait un enseignement de l'arabe accordant trop de place à la langue coranique : «ce sont ces poésies populaires dont quelques-unes méritent le nom de 'classiques', car depuis trois cents ans, elles forment le goût et la langue des indigènes. » (ibid. : XI)

Selon Messaoudi (2014), ce manuel réédité à plusieurs reprises, régulièrement donné en référence aux professeurs, invités à l'adapter en fonction des parlers et des usages locaux, restera un modèle inégalé jusqu'à l'Indépendance de l'Algérie.

\section{Manuels d'arabe et modèle français}

31 Tous les auteurs de manuels ne seront pas des enquêteurs « sociolinguistes » et certains publieront des manuels assez proches, dans la forme et le fond, des méthodes directes pour le français. Parmi ceux-ci figurent Ben Fatah (1904), Méthode directe pour l'enseignement de l'arabe parlé, Soualah (1904) Cours préparatoire d'arabe parlé. Enseignement par l'image et par la méthode directe et Abderrahman (1906), auteur de l'Enseignement de l'arabe parlé d'après la méthode directe. Lectures choisies.

Dans sa Préface, Ben Fatah qui fut directeur d'école arabe-française, rend un hommage appuyé à ses principaux inspirateurs :

Cette méthode que j'ai expérimentée depuis plusieurs années pour l'enseignement de l'arabe aux jeunes Européens, m'a été inspirée pour la Méthode et les procédés d'exercice de langage, de MM. Scheer et Mailhes, deux de mes anciens condisciples et amis de l'École normale, méthode qui a puissamment contribué à l'élaboration des programmes de l'Enseignement du français dans nos Écoles indigènes (1904: VII).

La méthode suit les programmes pour l'arabe arrêtés par le Rectorat où il est dit que "La méthode directe est inductive et pratique »: « elle prendra pour base la langue étrangère ", " elle partira de l'observation et non de l'abstraction »; " elle exercera l'élève à exprimer des idées au moyen du vocabulaire étudié »(ibid.: VIII). La transposition à l'enseignement de l'arabe des principes adoptés pour le français est manifeste : « Le maitre commencera par nommer distinctement l'objet qu'il désignera à l'attention de l'élève, puis celui-ci répètera lentement ce nom en s'efforçant de bien prononcer... Le musée scolaire pourra servir avantageusement à toutes ces premières leçons » (ibid. : VIII).

Le manuel de Ben Fatah ne comporte pas d'illustrations pour les exercices de langage de la première partie, s'appuyant sur les objets de la classe et du musée ou personnages de l'environnement, mais une dizaine de Causeries sur images clôturent les cours 
préparatoire et élémentaire avec des gravures originales d'Auguste Barbichon, auxquelles est associé le vocabulaire correspondant.

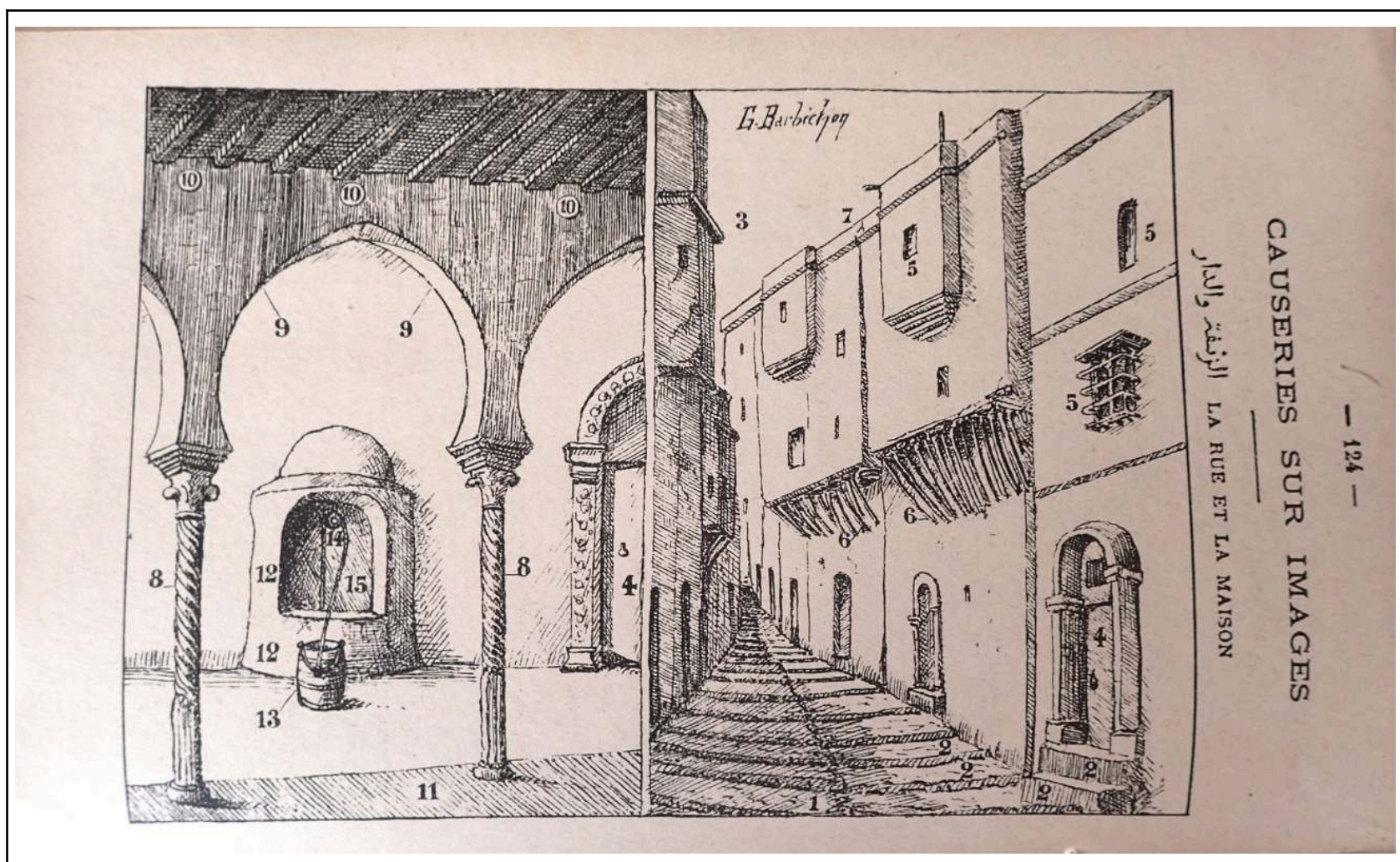

Fig. 1 : Ben Fatah, Méthode directe, 1904, p.124

Lorsque l'on ne peut plus s'appuyer sur la classe ou son environnement, « on recourt aux images. Signifiant et référent sont ainsi directement mis en relation. Les images vont [...] relayer le monde [...]» (Vigner $2018: 181)$.

Le Cours préparatoire d'arabe parlé. Enseignement par l'image et par la méthode directe (1904) de Mohamed Soualah est sans doute le plus représentatif de la MD, par sa méthode de langage reposant sur d'abondantes illustrations :

Les leçons doivent d'abord être orales; ainsi celles que je propose sont toutes concrètes. Le maitre doit les faire devant les élèves, suivant les indications du livre de l'élève et de la partie du maitre. Que l'instituteur songe avant tout: $1^{\circ}$ à faire pénétrer plusieurs fois tout ce qu'il dit; $2^{\circ}$ à faire accomplir les actions par les élèves. Après quoi le livre, tant par les gravures que par les légendes, servira de tableau complet et vivant de la leçon (Préface, IV-V). 


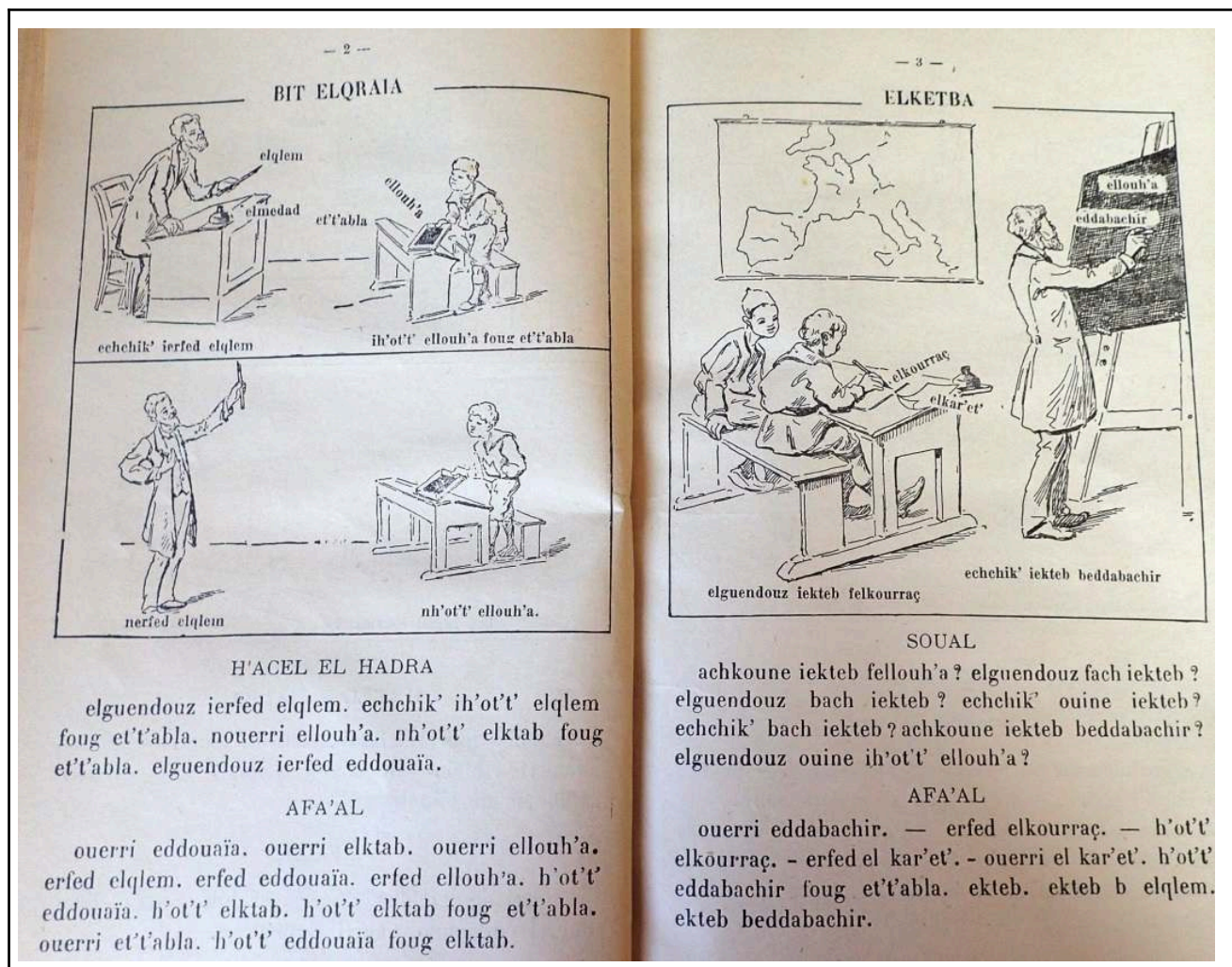

Fig. 2 - M. Soualah, Cours préparatoire d'arabe parlé, 1955, p. 2-3

La progression qui va du " connu à l'inconnu », du " proche au lointain », de la classe à l'école, à la maison, au douar, à l'habillement, aux voyages, etc. est caractéristique de la MD. Chaque leçon veille autant que possible à présenter une scène représentant le mode de vie européen et une scène le mode de vie algérien : 


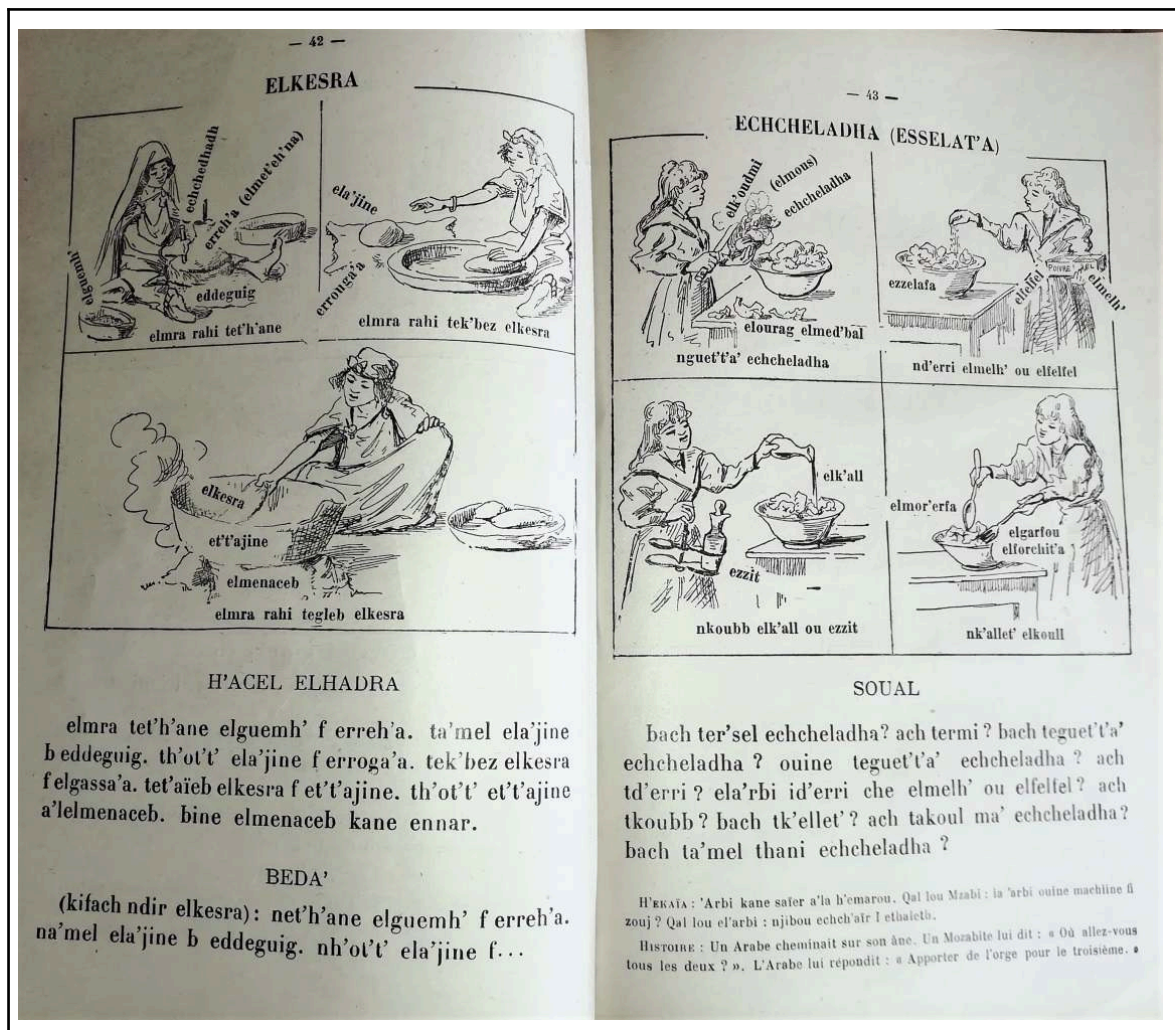

Fig. 3 - M. Soualah, Cours préparatoire d'arabe parlé, 1955, p. 42-43 régions, en « les soumettant au contrôle de ses élèves du Cours normal de Bouzareah » (Soualah 1955 : V). De même, il a opté pour les caractères latins identiques à ceux des livres de français: «Les enfants les apprendront mieux ainsi: de la sorte, l'enseignement de l'arabe deviendra un auxiliaire pour la lecture française. Plus tard au cours élémentaire [..] nous leur enseignerons les caractères arabes sans difficulté, tout en continuant les exercices de langage. » (Ibid.)

L'ouvrage comporte aussi des dialogues, un lexique, un memento avec des explications grammaticales et des corrigés d'exercice, il pourrait donc être utilisé sans maitre.

\section{La méthode directe et l'enseignement du kabyle : l'exemple de Boulifa}

Said Boulifa, instituteur, devenu professeur de langue kabyle à l'École normale et à l'École supérieure des Lettres d'Alger est le premier Kabyle à s'inscrire dans ce renouveau méthodologique: "Ses manuels scolaires constituent une référence incontestable pour ses contemporains et les générations ultérieures. Ils mettent en œuvre la méthode directe à partir de textes directement puisés auprès de locuteurs kabyles » (Sellès $2014: 142$ ).

Boulifa expose dans la préface de son premier manuel Une première année de langue kabyle. Dialecte Zouaoua (1910 [1897]), les objectifs de sa méthode :

Apprendre à parler et écrire correctement le kabyle, guider et encourager ceux qui veulent s'adonner à l'étude de cette langue, les mettre dans le moins de temps 
possible à même de converser avec les Indigènes, tel est le but que je me suis proposé en rédigeant ce modeste ouvrage qui n'est que le résumé des leçons que j'ai l'honneur de faire à M.M. les Elèves de la Section spéciale de l'Ecole normale d'AlgerBouzareah depuis décembre 1891 (Boulifa 1897 : Préface, I). Boulifa,
lecture :

Pour préparer le Kabylisant à la conversation, j'ai écarté ce genre de phrase que l'on ne trouve guère que dans les manuels et que l'on rencontre jamais dans la pratique ; je me suis efforcé de faire un choix judicieux de tournures et d'expressions qui sont d'un fréquent usage dans le langage courant (ibid. : II).

Le manuel de première année commence par un cours introductif sur l'alphabet et les adaptations nécessaires pour enseigner le kabyle. Chaque leçon se termine par des exercices de prononciation selon les instructions officielles de 1902. Puis les leçons procèdent selon une progression grammaticale (une règle avec exemples, remarques et exceptions) et lexicale selon les thèmes habituels (lexique de l'école, de la nourriture, $\mathrm{du}$ corps humain, de la famille, des animaux domestiques, sauvages, termes géographiques, le temps, les saisons, etc.). Ce premier manuel présente une structure assez semblable à celui de Ben Sedira (voir supra), Boulifa utilise le français pour introduire les leçons, seuls les exemples qui accompagnent les cours sont donnés en langue kabyle.

Dans la préface du Cours de deuxième année, étude linguistique et sociologique sur la Kabylie $d u$ Djurdjura, Boulifa souligne les limites de son premier manuel sur le plan du vocabulaire, car il ne contient que des noms usuels d'objets, de plantes et de personnes. Il décide d'éliminer toutes les leçons de grammaire, considérées comme acquises durant la première année et d'orienter le manuel vers la pratique orale de la langue, se rapprochant ainsi davantage de la $\mathrm{MD}$ : «Basée sur la conversation, la méthode n’a, en effet, d'autre but que d'habituer l'élève à la manœuvre pratique, à l'emploi, avec ses propres moyens, des connaissances acquises pour l'amener graduellement à s'initier à la gymnastique spéciale du langage. » (Boulifa 1913 : VIII)

Les multiples sujets de la vie pratique vont constituer la base des cours:

Pour le kabyle, il n'y a pas de littérature [...] il convient [...] de pouvoir s'exprimer avec les termes simples et connus de tout le monde, de connaitre et d'employer le même langage que l'artisan, le commerçant [...] Partant de ce principe, nous estimons donc que l'étude de la vie pratique de ceux dont on veut connaître la langue, est là, toute trouvée pour nous servir de base et pour nous procurer toutes les matières voulues, devant composer notre Deuxième année (ibid. : IX).

Pour rendre la méthode attrayante, tous les sujets sont abordés sous forme de dialogues, où le personnage principal, un Français nommé Afrensis, ayant le vif désir de s'instruire, interrogera les gens pour les connaitre et se familiariser avec les coutumes de ce pays: «Connu et aimé de tous, il lui sera loisible de tout voir et tout entendre, sans crainte d'indiscrétion, il pourra discuter sur la vie intime du kabyle qui lui sera dévoilée dans toute sa réalité » (ibid. $\mathrm{X}$ ) 


\section{Afrensis. - A'slamak! anda theddith akka çebah'a? stheq'sar' fell ak r'er oukhkham,oufir' oulach ik. AQ'BAïLI. - Tsid'ets, oulach ii çebah'a; roh'er' ebbon- Wher' armi d'aa'rq'oub. \\ - I themeddith a, ach ard thekhd'emedh? as-ed ma thesthoufadh anneq'eçcer kra louah'id'. \\ - Iouq'em, r'ef achl'al essa'a ara d our'aler' ? \\ - Thoura ad'a'ddir', ad'efdherer', ou tsa't't'il ara, aq'lii ak erdjour' annesoû akken lq'ahoua.}

Fig. 4 - Boulifa (1913 :1). Méthode de langue Kabyle. Cours de deuxième année.

La méthodologie directe mise en œuvre par Boulifa est originale. Pour ce professeur de l'École normale, enseigner une langue c'est «satisfaire à la fois la linguistique et la sociologie » (ibid. X) :

connaître une langue c'est connaître la mentalité, l'esprit, le génie de ceux qui parlent cette langue ; or, la mentalité d'un groupe quelconque d'individus, ne peut être pénétrée, saisie, comprise que par une étude approfondie, une connaissance exacte de leur caractère et de leurs mœurs. C'est là, pensons-nous, l'esprit de la méthode directe (ibid. IX).

Cette définition donne à l'enseignement une dimension culturelle et sociale et l'éloigne de la visée pratique de "communication » assignée à la MD, parti pris contre lequel se sont élevés beaucoup de pédagogues dans les régions françaises où la MD menaçait l'usage scolaire des langues parlées (Cortier \& Puren 2008). Le manuel ne comporte pas d'illustrations, son public est constitué d'élèves-maîtres qui pouvaient se passer facilement des illustrations pour saisir le sens des leçons. Cette «description fine des coutumes kabyles du Djurdjura reste[ra] jusque dans les années 1960 le principal manuel en usage pour l'étude du berbère en France et en Algérie » (Sellès 2014 : 143).

Boulifa a exploité des textes culturels, anthropologiques ou ethnographiques, comme supports, dans une vision didactique moderne, où l'enseignement institue l'importance de la tradition orale «dans les différents processus de l'élaboration didactique des langues minoritaires, mêlant ainsi très souvent les avancées les plus significatives en matière de méthodes et les savoir-faire ancestraux de la transmission orale » (Di Meglio $2015: 6)$. 


\section{Conclusion}

51 Cette étude a ouvert pour nous une entrée originale dans l'enseignement des langues en Algérie et nous a permis de revisiter les connaissances historiques et didactiques sur cette période. Les enjeux politiques liés à l'enseignement des langues y sont clairement exposés, qu'il s'agisse du français, de l'arabe, ou du kabyle. Les exercices de langage, les «activités orales » caractéristiques de la MD se trouvent à la base de l'enseignement, augurant ainsi une méthodologie didactique active, dite "pratique» (Cortier 1999; Vigner 2019), à la fois distincte de celle de la métropole et semblable à elle. Comme dans l'enseignement primaire de la III ${ }^{e}$ République, il s'agit de partir de ce que l'enfant connaît, d'aller du connu pour aller vers le moins connu ou l'inconnu, du concret vers l'abstrait, du particulier vers le plus lointain en s'appuyant sur le musée scolaire et la leçon de choses. Dans l'esprit des éducateurs républicains, le local est un préalable au national dans l'ordre de la connaissance. Mais pour les plus grands élèves ou les adultes, l'inadéquation et les insuffisances de la méthode directe, critiquées dans de nombreuses régions françaises et au sein même de la Revue pédagogique, seront contournées par des auteurs comme Desparmet et Boulifa, qui vont tirer parti des collectes de terrain encouragées par l'École supérieure d'Alger et souvent financées par le ministère de l'Instruction publique pour alimenter leurs manuels et créer des textes originaux pour l'enseignement-apprentissage, qui contribueront à renforcer les identités régionales. Ces manuels ont ainsi accumulé un corpus important de documents ethnographiques dans l'intérêt des langues et cultures autochtones.

De même, plusieurs instituteurs indigènes et européens formés à l'École normale d'instituteurs de Bouzareah en arabe et en berbère par la MD et qui suivirent aussi les cours de Basset à l'université d'Alger ont réalisé leurs premiers travaux linguistiques en Algérie, avant de donner un nouvel essor à leurs études au $\mathrm{Maroc}^{7}$, œuvrant ainsi pour la constitution de langues à dimension maghrébine. Des avancées pour la plupart sans lendemain... Aujourd'hui on lutte en Algérie et au Maroc pour la reconnaissance de ces langues et leur utilisation dans l'enseignement, alors que ce sont les langues maternelles et d'usage des populations. En effet, les mouvements nationalistes ont manifesté dès 1920 leur « opposition à l'enseignement des parlers, au nom d'une langue littéraire, véhicule de la tradition musulmane et support d'une identité nationale arabe » (Messaoudi $2014: 104)$ et de ce fait contribué à exclure ces variétés parlées de l'enseignement.

\section{BIBLIOGRAPHIE}

\section{Sources primaires}

ABDERRAHMAN, Mohammed (1906). Enseignement de l'arabe parlé \& de l'arabe régulier d'après la méthode directe. Lectures choisies. Alger : Jourdan. En ligne : [https://archive.org/details/ 
enseignement

dela00abda/page/n178/mode/2up].

FATAH, Brahim (1904). Méthode directe pour l'enseignement de l'arabe parlé. Aux nouveaux programmes. Cours élémentaire moyen et supérieur. Alger : Jourdan.

BEN SEDIRA, Belkassem (1900 [1875]). Le Cours pratique de langue arabe. Exercices d'écriture, de lecture et de mémoire : règles du langage, thèmes et versions; dialogues variés, contes amusants, lettres familières. Alger : Jourdan.

BEN SEDIRA, Belkassem ( ${ }^{5} 1910$ [1886]). Dictionnaire français-arabe de la langue parlée en Algérie. Alger : Jourdan.

BEN SEDIRA, Belkassem (1887). Cours de langue kabyle, Grammaire et versions, précédé d'Une mission en Kabylie. Alger : Jourdan. En ligne : [https://cinumed.mmsh.univ-aix.fr/collection/item/16860cours-de-langue-kabyle-grammaire-et-versions?offset=7].

BUISSON, Ferdinand (1887). « Nos pionniers en Afrique ». Revue pédagogique, 10, 481-512.

BUISSON, Ferdinand (dir.) (1887). Dictionnaire de pédagogie et d'instruction primaire. Partie 1, t.1. En ligne : [https://gallica.bnf.fr/ark:/12148/bpt6k24232h.texteImage].

BOULIFA, Said. (1910 [1897]). Une première année de langue kabyle (dialecte zouaoua). À l'usage des candidats à la prime et au brevet de kabyle. Alger : Jourdan. En ligne : [https://www.fichier-pdf.fr/ 2015/02/18/boulifa-si-a-1897-une-premiere-annee-de-langue-kabyle-dialecte-zouaoua-alger-eda-jourdan/boulifa-si-a-1897-une-premiere-annee-de-langue-kabyle-dialecte-zouaoua-alger-ed-ajourdan.pdf].

BOULIFA, said (1913). Méthode de langue kabyle (cours de deuxième année). Étude linguistique, sociologique sur la Kabylie du Djurdjura. Texte zouaoua, suivi d'un glossaire. Alger : Jourdan. En ligne : [https:// www.fichier-pdf.fr/2014/01/24/111156855-methode-de-la-langue-kabyle-cours-de-deuxiemeannee-s-a-boulifa-1913/111156855-methode-de-la-langue-kabyle-cours-de-deuxieme-annee-s-aboulifa-1913.pdf].

CARRÉ, Irénée (1889). Méthode pratique de langage, de lecture, de calcul, etc. plus spécialement destinée aux enfants des provinces où l'on ne parle pas français, et qui arrivent en classe ne comprenant ni ne sachant parler la langue nationale. Paris : Colin.

CARRÉ, Irénée (1891). « De la manière d'enseigner les premiers éléments du français aux indigènes, dans nos colonies et dans les pays soumis à notre protectorat ». Revue Pédagogique, Tome XVII, 4 , 289-314.

DESPARMET, Joseph (1907 [1904]). Enseignement de l'arabe dialectal d'après la méthode directe. Première période : vocabulaire et lectures. Seconde période et brevet d'arabe. Textes de lecture : coutumes, institutions, croyances. Blida : Mauguin.

JEANMAIRE, charles (1911). «Algérie ». In Ferdinand BUISSON (dir.) Nouveau dictionnaire de pédagogie et d'instruction primaire. En ligne : [http://www.inrp.fr/edition-electronique/lodel/dictionnaireferdinand-buisson/document.php?id=2012].

MACHUEL, Louis (1875). Méthode pour l'étude de l'arabe parlé (idiome algérien). Alger : Jourdan.

(1877). Première année d'arabe, à l'usage des classes élémentaires du lycée, des collèges, des écoles primaires, etc. etc. Alger : Jourdan. $2^{\mathrm{e}}$ édition en ligne : [https://gallica.bnf.fr/ark:/12148/ bpt6k1163269p/f9.item]. 
PERRIN, Alfred (1894). « De la manière d'enseigner le français aux indigènes d'Algérie et de Tunisie ». Revue pédagogique, 24 : 107-122. En ligne : [https://education.persee.fr/docAsPDF/ revpe_2021-4111_1894_num_24_1_3660.pdf].

MUSÉE PÉDAGOGIQUE (1890). Mémoires et documents scolaires publiés par le musée pédagogique. Plan d'études et programmes de l'enseignement primaire des indigènes en Algérie, fascicule $\mathrm{n}^{\circ} 114$. Alger : Adolphe Jourdan ; Paris : Hachette, Delagrave, Delalain, Picard, A. Colin, Alc. Picard et Kaan Éditeurs. En ligne : [https://gallica.bnf.fr/ark:/12148/bpt6k5496942r.texteImage].

POULARD, Maurice (1910). L'enseignement pour les indigènes en Algérie. Alger : imp. Administrative. RAMBAUD, Alfred (1892). «L'Enseignement primaire chez les indigènes musulmans d'Algérie et notamment dans la Grande-Kabylie ». Revue Pédagogique, $\mathrm{n}^{\circ} 11,15 / 11 / 1891, \mathrm{n}^{\circ} 12,15 / 12 / 1891, \mathrm{n}^{\circ} 1$, 15/01/1892, n² 15/02/1892, (de novembre 1891 à février 1892).

RAMBAUD, Alfred (1887). « Nos pionniers en Afrique ». Revue pédagogique, n6, 15 juin 1887, 80-511. SCHEER, Jean-Eugène (1892). Projets de construction d'écoles indigènes en Kabylie, rapport de M. Scheer, inspecteur des écoles indigènes. Alger : imp. Adolphe Jordan.

SOUALAH, Mohammed ( ${ }^{4} 1955$ [1904]). Cours préparatoire d'arabe parlé. Enseignement par l'image et la méthode directe sans caractères arabes (conforme aux programmes officiels). Illustrations de Herzig. Alger : Jourdan.

\section{Sources secondaires}

BERDOUS, Nadia (2017). La question du kabyle en Algérie. Individuation sociolinguistique et processus d'élaboration didactique. Thèse de doctorat, Université de Corte.

BISQUERRA, claude (2001). «Les écoles indigènes en Algérie à la fin du XIX siècle : l'expérience de maitres français et indigènes dans le sud-est algérien ». Documents pour l'histoire du français langue étrangère ou seconde, 27, 155-172.

CORTIER, claude (1998). Institution de l'Alliance française et émergence de la francophonie. Politiques linguistiques et éducatives. 1880-1914. Thèse de doctorat, Université Lyon 2.

CORTIER, claude (1999). « Il y a un siècle...l'enseignement pratique à l'Alliance française ». Mots. Les langages du politique, 61, 127-136.

CORTIER, claude \& PUREN, Laurent (2008). « Français et langues régionales et/ou minoritaires : une mise en convergence difficultueuse ». Repères, 38, 63-80.

DI MEGLIO, Alain (2015). « Didactique des langues minoritaires et enjeux de légitimation ». In Philippe BLANCHET \& Patrick CHARDENET (dir.), Guide pour la recherche en didactique des langues et cultures (approches contextualisées). Paris : Éditions des archives contemporaines, 325- 333.

LARZUL, sylvette (2010). « Grammatisation et lexicographie de l'arabe algérien au XIX ${ }^{\mathrm{e}}$ siècle ». Synergies Monde arabe, 7, 89-100. En ligne : [https://gerflint.fr/Base/Mondearabe7/larzul.pdf].

LARZUL, sylvette (2014). «Les manuels de langue arabe des débuts de l'Algérie coloniale (1830-1871) ». In Sylvette LARZUL \& Alain MESSAOUDI (éd.), Manuels d'arabe d'hier et d'aujourd'hui. France et Maghreb, XIX ${ }^{e}-X X I^{e}$ siècle. Paris : Éditions de la Bibliothèque nationale de France LARZUL, sylvette \& MESSAOUDI, Alain (2014). Manuels d'arabe d'hier et d'aujourd'hui. France et Maghreb, $\mathrm{XIX}^{e}-\mathrm{XXI}{ }^{e}$ siècle, Les colloques de la BNF. Paris : Éditions de la Bibliothèque nationale de France. En 
ligne : [https://books.openedition.org/editionsbnf/

233?lang=de].

LIEUTARD, Hervé \& VERNY, Marie-Jeanne (2007). L'école française et les langues régionales XIXe et XXe siècles. Montpellier : P.U.M.

MARIET, François (1978). «Idéologie scolaire et culture en Algérie. À propos de quelques ouvrages récents consacrés à l'Algérie ». Revue française de sociologie, 19-3, 427-434. En ligne : [https:// www.persee.fr/doc/rfsoc_0035-2969_1978_num_19_3_6917].

MESSAOUDI, Alain (2014). « Progrès de la science, développement de l'enseignement secondaire et affirmation d'une 'méthode directe' (1871-1930) ». In Sylvette LARZUL \& Alain MESSAOUDI (éd.), Manuels d'arabe d'hier et d'aujourd'hui. France et Maghreb, XIX ${ }^{e}-X X I^{e}$ siècle. Paris : Éditions de la Bibliothèque nationale de France. En ligne : [https://books.openedition.org/editionsbnf/247? lang=de].

MESSAOUDI, Alain (2015). Les arabisants et la France coloniale. 1780-1930. ENS Editions. En ligne :

[https://books.openedition.org/

enseditions/3705?lang=de].

PUREN, Christian (1988). Histoire des méthodologies de l'enseignement des langues. Paris : CLE international.

PUREN, Laurent (2004). L'école française face à l'élève alloglotte. Contributions à une étude des politiques linguistiques éducatives mises en cuvre à l'égard des minorités linguistiques scolarisées dans le système éducatiffrançais du XIXe siècle à nos jours. Thèse de doctorat en Didactologie des Langues et des Cultures, Université Paris III.

SELLES, Michèle (2014). « Les manuels de berbère publiés en France et en Algérie (XVIII ${ }^{\mathrm{e}}$-XX siècle) : d'une production orientaliste à l'affirmation d'une identité postcoloniale ». In Sylvette LARZUL \& Alain MESSAOUDI (éd.), Manuels d'arabe d'hier et d'aujourd'hui. France et Maghreb, XIXe-XXIe siècle. Paris : Éditions de la Bibliothèque nationale de France. En ligne : [https:// books.openedition.org/editionsbnf/266?lang=de].

VIGNER, Gérard (2018). « Leçons de mots, leçons de choses ; vocabulaire, langage et connaissances $\mathrm{du}$ monde dans les approches du français à l'école coloniale ». Documents pour l'histoire du français langue étrangère ou seconde, 60-61, 173-194.

VIGNER, Gérard (2019). « Les exercices de langage : du Plan d'Études et programmes de l'enseignement indigène en Algérie au Bulletin de l'enseignement indigène de l'Académie d'Alger (1893-1914) ». Documents pour l'histoire du français langue étrangère ou seconde, 62-63, 403-428.

\section{NOTES}

1. Ce développement sera cependant très limité. Cf. Kadri, A. (2007) "Histoire du système d'enseignement colonial en Algérie ». In Frédéric Abécassis et al. La France et l'Algérie: leçons d'histoire : De l'école en situation coloniale à l'enseignement du fait colonial. Lyon : ENS Éditions, 19-39.

2. Des Cours normaux furent aussi annexés aux écoles normales d'instituteurs d'Alger et de Constantine.

3. 36 écoles ont été répertoriées en 1870 .

4. Plusieurs travaux dont Puren (2004:208-210) montrent comment la méthode dite maternelle a été introduite en France via l'Alsace ou la Lorraine, les enseignants alsaciens ayant été initiés à la méthode « intuitive ou maternelle » au contact des pédagogues allemands. 
5. Notamment Auguste Cherbonneau, partisan de la création d'un arabe algérien moderne basé sur le dialecte, auteur d'un Dictionnaire français-arabe pour la conversation en Algérie (1872) (Larzul 2010 : 95).

6. Titre du recueil de textes qui constitue la seconde partie de l'ouvrage.

7. C'est le cas d'Edmond Destaing, d'Émile Laoust, de Mohammed Nehlil ou de Mohammed Abès (Sellès, $2014:$ 141).

\section{RÉSUMÉS}

Cet article s'intéresse aux développements de l'Instruction publique introduits en Algérie à partir de 1883. L'enseignement du français par des méthodes privilégiant l'école de langage et la méthode directe, recommandée par les programmes, va prendre une place prépondérante, dans les écoles pour Européens ou "Indigènes ». Un enseignement restreint de l'arabe parlé sera maintenu dans toutes les écoles. Des primes d'encouragement viendront conforter l'implication des instituteurs titulaires d'un brevet en langue arabe et/ou kabyle, afin qu'ils puissent en milieu rural développer des échanges avec les populations. Des méthodes et manuels originaux, souvent innovants, basés sur des recueils de langue parlée et des documents ethnographiques seront créés pour l'enseignement de ces langues aux Européens qui attestent aujourd'hui d'une vitalité didactique propre à cette période.

This paper deals with the developments of Public Instruction introduced in Algeria from 1883. The teaching of French by methods favoring the language spoken and the direct method, recommended by the programs, will take a preponderant place, in schools for Europeans or schools for "Indigenous people". Restricted teaching of spoken Arabic will be maintained in all schools. Bonuses and incentives will reinforce the involvement of teachers with certificate or diplomas in Arabic and/or Kabyle language, so that they can develop exchanges with the populations, in rural areas. Original and innovative manuals based on spoken language collections and ethnographic documents will be developed for teaching these languages to Europeans. They attest today to a didactic vitality specific to this period.

\section{INDEX}

Keywords : Algérie, politiques coloniales, méthode directe, manuels de langue française, langue arabe, langue kabyle

Mots-clés : Algérie, politiques coloniales, méthode directe, manuels de langue française, langue arabe, langue kabyle

\section{AUTEURS}

\section{NADIA BERDOUS}

Université de Bouira - n_berdous@yahoo.fr 


\section{CLAUDE CORTIER}

UMR ICAR, Université de Lyon - claude.cortier@gmail.com 